\title{
FORM, Diagrams and Topologies
}

Franz Herzog, ${ }^{a}$ Ben Ruijl, ${ }^{a b}$ Takahiro Ueda, ${ }^{a}$ J.A.M. Vermaseren*, ${ }^{*}$ Andreas Vogt, ${ }^{c}$ a Nikhef, Amsterdam

$b$ Leiden University

c University of Liverpool

E-mail: fherzog@nikhef.nl, benruyl@gmail.com, tueda@nikhef.nl, t68@nikhef.nl, Andreas.Vogt@liverpool.ac.uk

We discuss a number of FORM features that are essential in the automatic processing of very large numbers of diagrams as used in the Forcer program for 4-loop massless propagator diagrams. Most of these features are new.

Loops and Legs in Quantum Field Theory - LL 2016,

25 April - 29 April 2014

Leipzig, Germany

${ }^{*}$ Speaker.

(C) Copyright owned by the author(s) under the terms of the Creative Commons 


\section{Introduction}

When we do a higher order calculation in field theory roughly 5 steps are involved:

\section{Diagram generation}

Notation Bring diagrams to a notation that is compatible with the libraries.

Physics Projections, Feynman rules, expansions, rearrangements, etc.

Reduction Express everything in terms of master integrals.

Integration Work out the master integral.

Here we will concentrate on the second step and some aspects of the fourth step as encountered in the Forcer program [1]. For the first step we use QGRAF [2], we use Forcer in the fourth step and we take the master integrals from the papers by Baikov and Chetyrkin [3], and Lee, Smirnov and Smirnov [4]. Of course we use Form [5, 6] throughout.

The third step is in principle trivial (unless you are into IR rearrangements and $R^{\star}$ operations), but if one likes to use gauge parameters and lots of gluons, it is possible to make some essential optimizations.

With quarks Ward identities can simplify things. For the gluons the key point is to notice that the canonical representation of the vertex

$$
V^{\mu \nu \rho}\left(p_{1}, p_{2}, p_{3}\right)=\left(p_{1}^{\rho}-p_{2}^{\rho}\right) \delta^{\mu \nu}-\left(p_{2}^{\mu}-p_{3}^{\mu}\right) \delta^{\nu \rho}-\left(p_{3}^{\nu}-p_{1}^{\nu}\right) \delta^{\rho \mu}
$$

is rarely the most efficient. Consider what happens when one or more indices are contracted with 'its own vector':

$$
\begin{aligned}
V^{\mu \nu \rho}\left(p_{1}, p_{2}, p_{3}\right) p_{3}^{\rho} & =\left(p_{2} \cdot p_{2}-p_{1} \cdot p_{1}\right) \delta^{\mu \nu}+p_{1}^{\mu} p_{1}^{\nu}-p_{2}^{\mu} p_{2}^{\nu} \\
V^{\mu \nu \rho}\left(p_{1}, p_{2}, p_{3}\right) p_{1}^{\mu} p_{2}^{\nu} & =p_{1}^{\mu} p_{1} \cdot p_{2}-p_{2}^{\mu} p_{1} \cdot p_{1} \\
V^{\mu \nu \rho}\left(p_{1}, p_{2}, p_{3}\right) p_{1}^{\mu} p_{2}^{\nu} p_{3}^{\rho} & =0
\end{aligned}
$$

You can see an obvious improvement in the number of terms and the killing of denominators. Additionally many terms pass vectors with 'their own index' on to the next vertex, leading to more simplifications. To start this game it is best to rewrite the triple gluon vertex as

$$
V^{\mu \nu \rho}\left(p_{1}, p_{2}, p_{3}\right)=\left(2 p_{1}^{\rho}+p_{3}^{\rho}\right) \delta^{\mu \nu}-\left(2 p_{2}^{\mu}+p_{1}^{\mu}\right) \delta^{\nu \rho}-\left(2 p_{3}^{\nu}+p_{2}^{\nu}\right) \delta^{\rho \mu}
$$

or a different rewrite (it is not unique) in a way that three vectors go with their own index. Starting substitutions with one such vertex and then each time substituting the vertex with the largest number of 'autocontractions' gives a very big savings on the purely gluonic diagrams. When gauge parameters are involved this is even more obvious.

We will start with concentrating on step two. In past work that involved DIS $[7,8]$ we had a program that brought the diagrams to the needed notations, determined the topology, the color factors, symmetries, etc. We called this program convdia.frm as opposed 
to calcdia.frm that does the steps three and four (and five). The whole was managed by a database program with make-like facilities called minos (also available for free from the FORM website).

The first version of convdia.frm was made by Paulo Nogueira [7] when he was staying at Nikhef for a year in the mid-1990's. After that it underwent many changes. Hence Paulo may not recognize any of it. The development inspired changes in Form as well.

Because there is no good reason to change the above setup, we kept this line of approach, but reprogrammed most of it. In this talk we will go over some of the main steps, their subtleties and some of the recent changes in Form.

Next we look at a recent function in Form that introduces a new concept in symbolic manipulation.

Finally we make some remarks about expansions in $\epsilon=2-D / 2$.

This work is part of the HEPGAME project which is supported by the ERC advanced grant 320651.

\section{Insertions}

In this step we collect the diagrams which together form sub-propagators as insertion graphs. This means that we will have lines in which the power of the denominator is not an integer. This gives great savings in the calculation. Take for instance the diagram

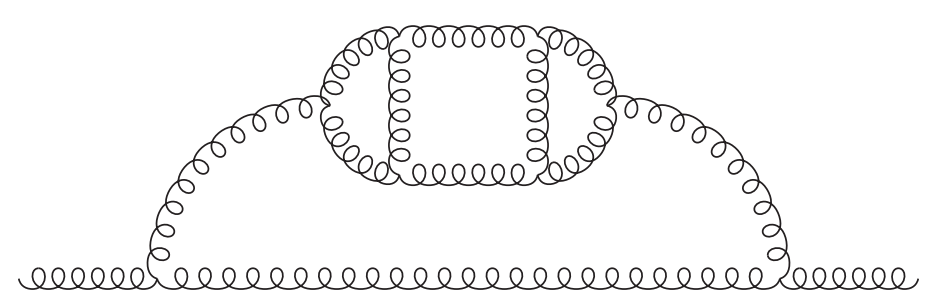

This diagram is part of a one loop diagram in which one line is a three loop gluon propagator. Such a 3-loop propagator contains actually many diagrams, 254 to be precise, and is relatively expensive to compute.

In addition it comes back in other calculations as in

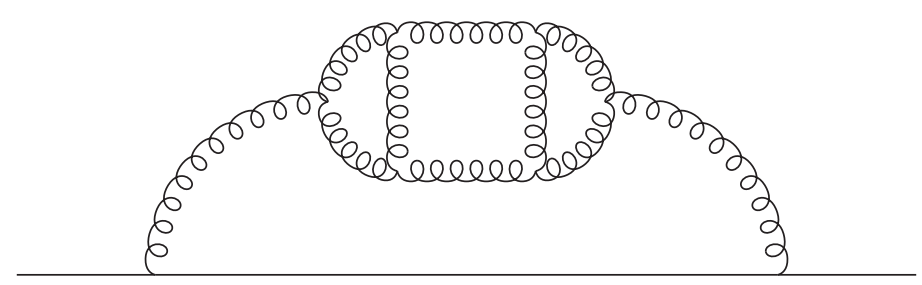

or in the computation of splitting functions and coefficient functions: 


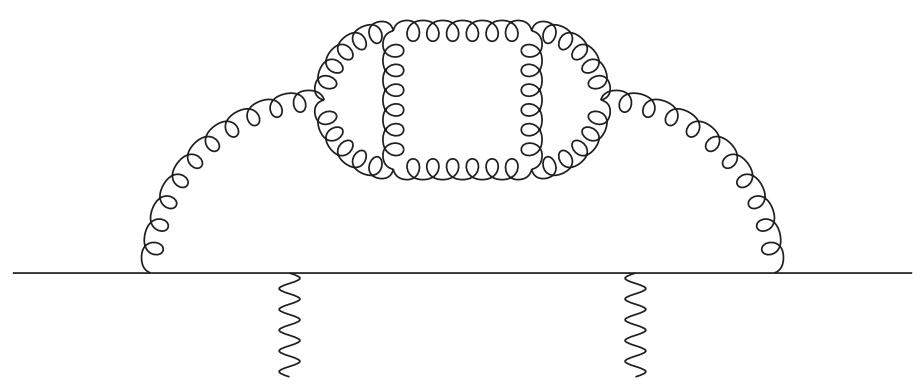

Hence it is a good idea to compute all possible propagators to a sufficient number of loops in terms of master integrals and rational coefficients. This way we are guaranteed to have sufficient accuracy by the time we have to add things. And now we have to compute only the diagrams
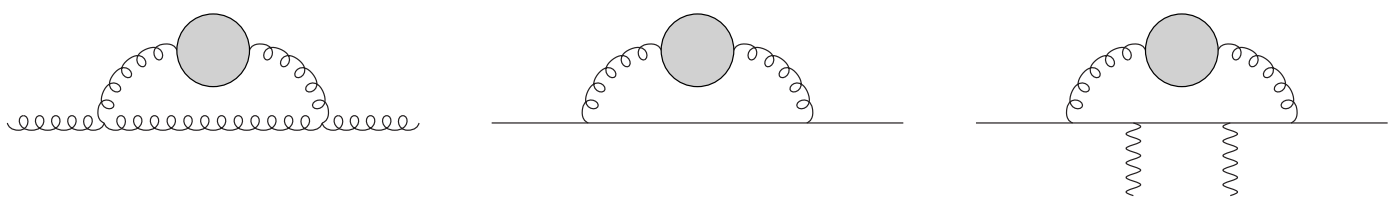

It does mean however that our master integrals will look a little bit different from the ones in the literature:
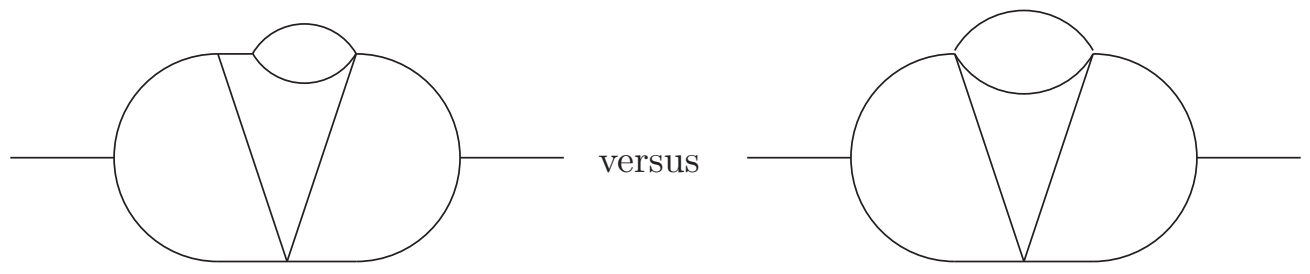

The conversion is of course simple. Put the right diagram through Forcer and it expresses the master from the literature in terms of our masters. Do this for all of them and invert the relations.

Actually one can use this technique also in the Reduze [11, 12] approach by backsubstituting the complete propagator in terms of its master integrals into the diagram. This could save Reduze enormous amounts of work.

At this point we have only one problem: how to collect all diagrams that form a single effective propagator? It is not easy to get QGRAF to do this [13]. In our case we do this in FORM.

We first select a unique representative diagram that belongs to each propagator. These are for the 1-loop, 2-loop, 3-loop gluon propagators (don't forget the minus sign of the ghost loop): 


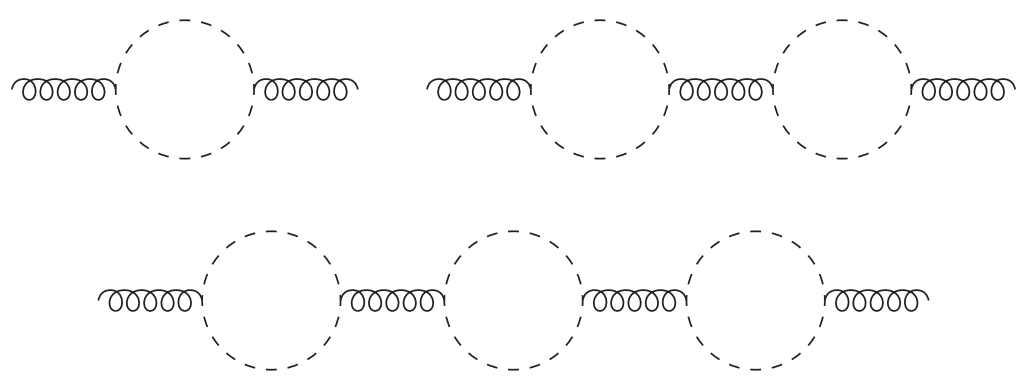

For the quark and ghost propagators we have similar representatives. Once we have isolated the representatives we have to throw away all other two-point sub-diagrams. The technical question is how to do this. In the intuitive way, one has to do some pretty fancy pattern matching and the more loops there can be, the more complicated the patterns can become and the larger their number. This is how it was done in the previous versions of convdia.

For a better solution we will make use of a new FoRM feature that was actually developed for something different: graph automorphisms. We are referring to a new option for the id-statement that generates all different matches in the pattern matcher. This option is the "all" option. A very simple example is:

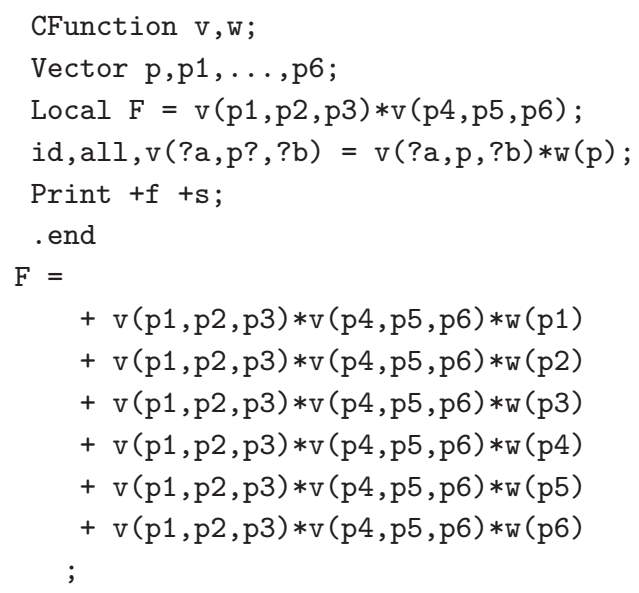

Now let us see how we will use that here. Assume that we have a lot of diagrams like

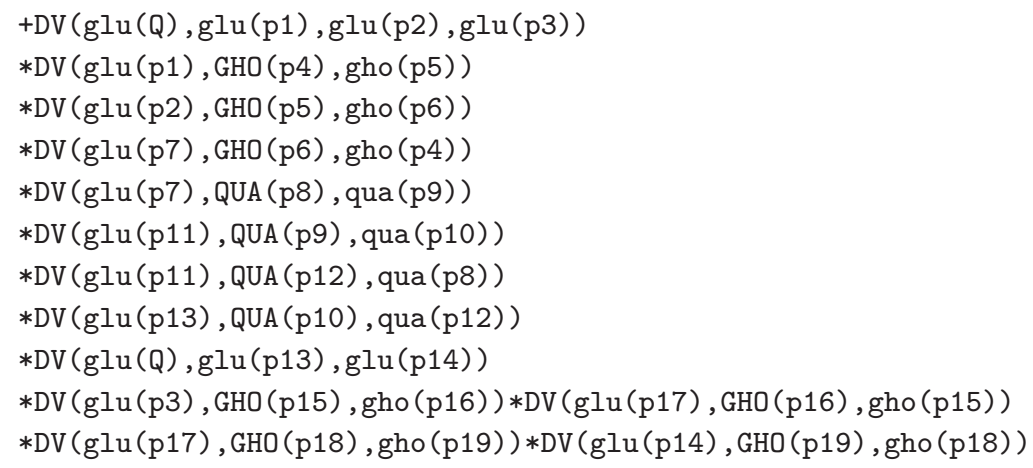

which looks like 


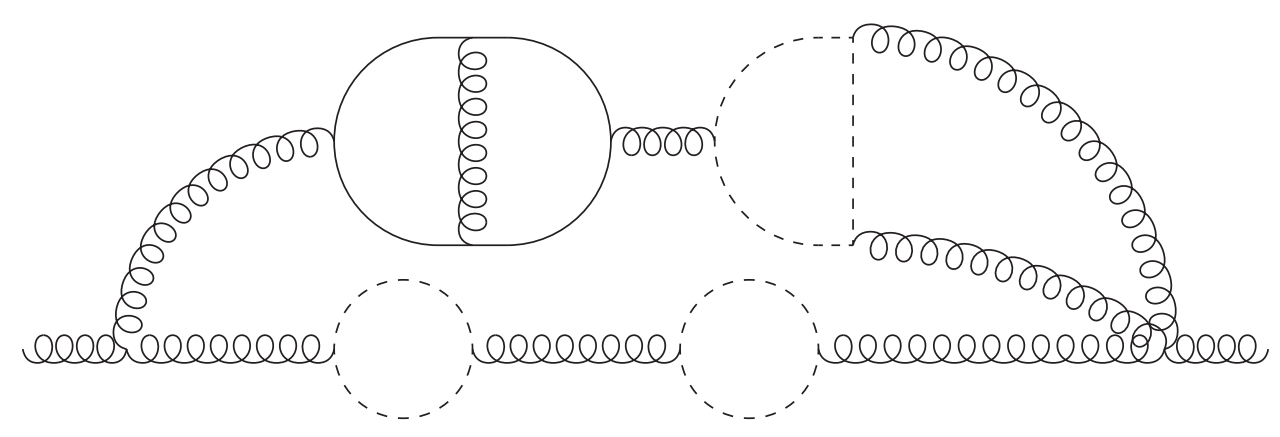

We start with taking out our representatives:

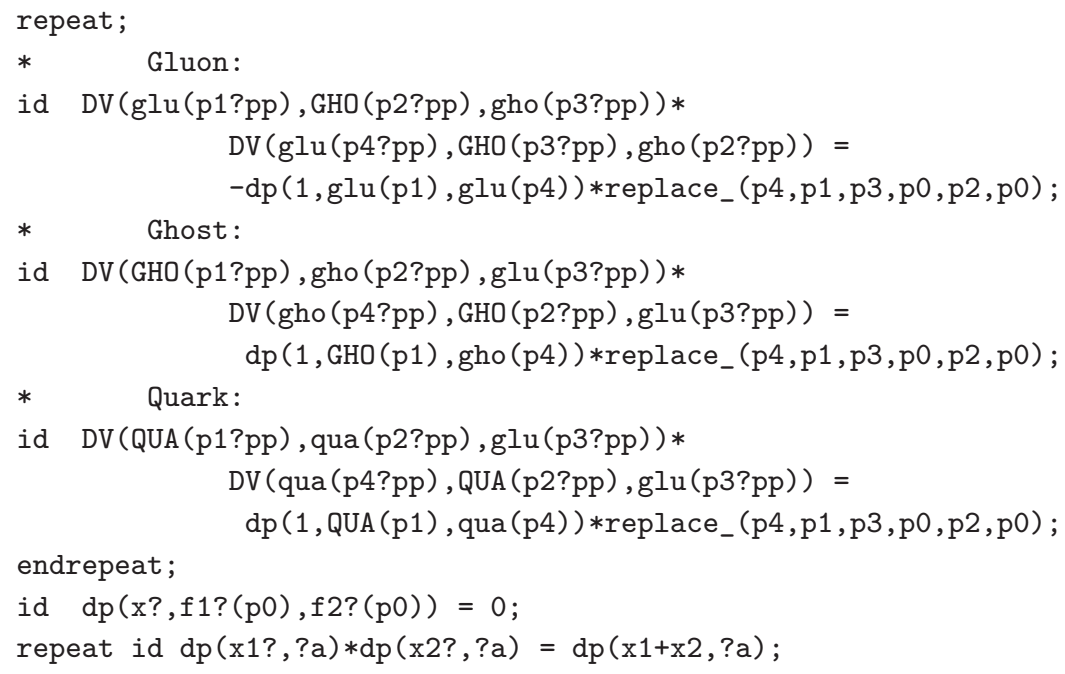

At this point we have a propagator function that tells how many loops are in it, what particles and what momentum. Our example diagram becomes now
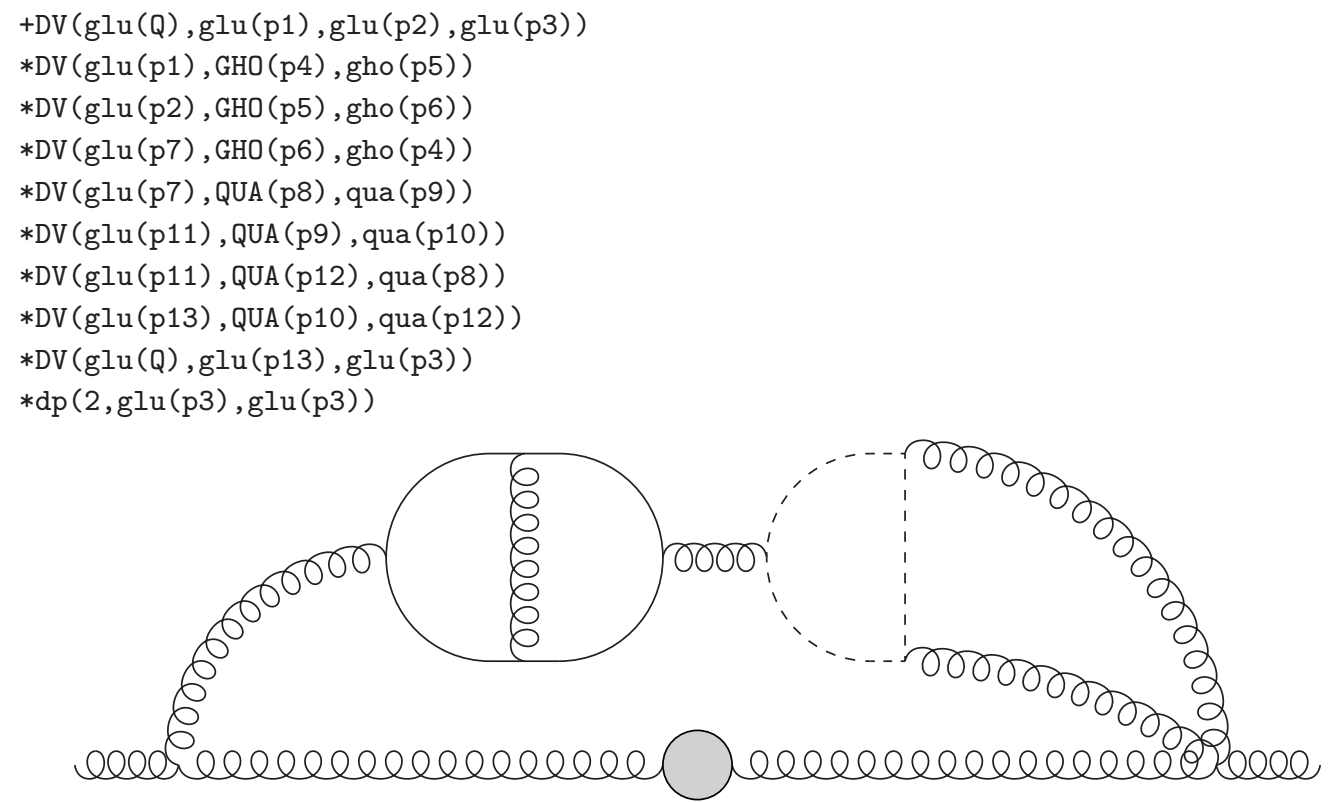
Now we have to eliminate all remaining diagrams that have a two-point function inside. For this we make a copy of our vertices in which we only put the momenta in the copy, and we collect these copied vertices in a function acc.

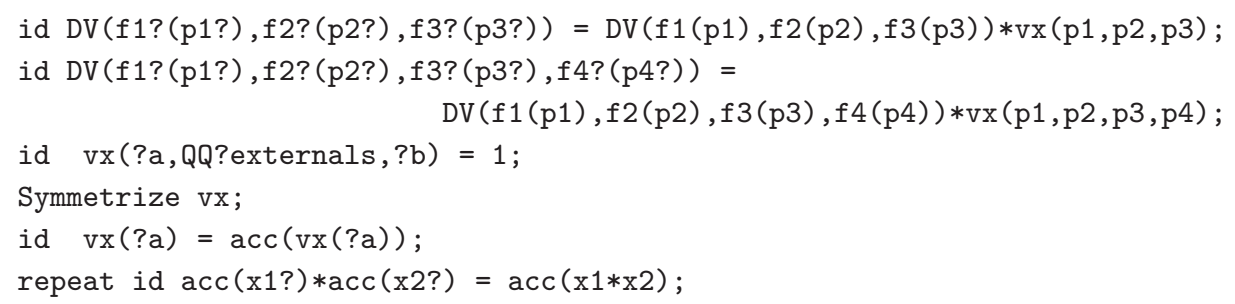

Notice that we eliminate the vertices with external lines because they can never be part of an internal propagator. We obtain:

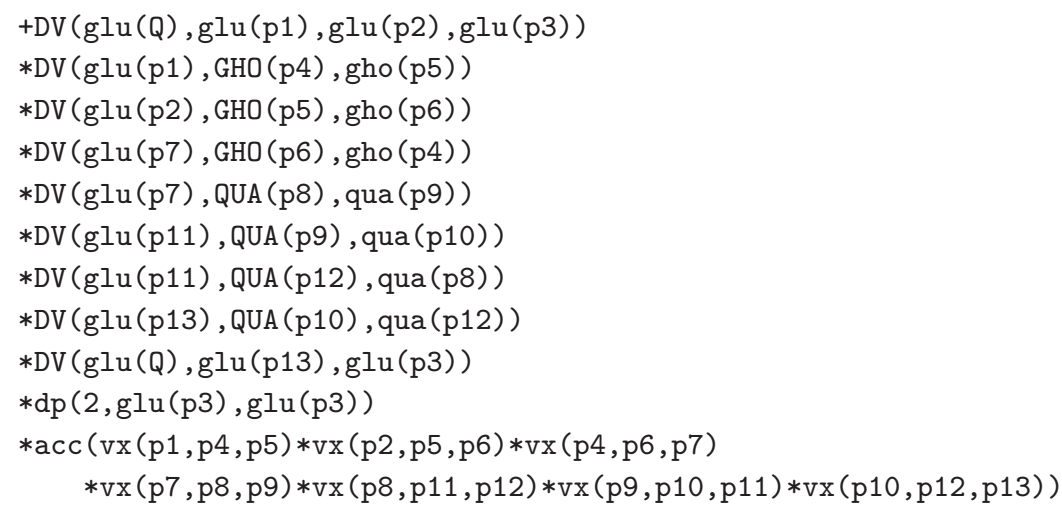

Now comes the algorithm: starting from each vertex, we construct the collection of vertices that are connected to it. We add them one by one and if at any time the total number of open lines of such a group is 2, this group is part of a propagator and we can eliminate the diagram. We have to try this in all possible ways.

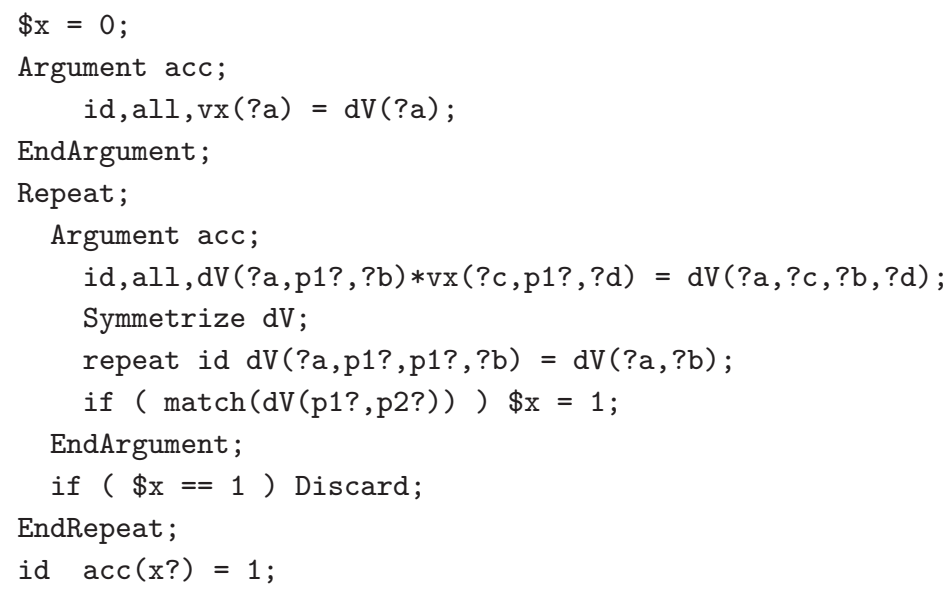

In the beginning we take one vx in all possible ways. This means that if there are 7 vertices, we generate 7 terms inside acc, each of which has one (different) vx replaced by $\mathrm{dV}$. Then in the repeat we add, in all possible ways each time one vx to the $\mathrm{dV}$ and 
eliminate repeated vectors, If at any time any of the terms inside acc has a dV with only two arguments we set a mark inside $\$ \mathrm{x}$ and the whole term can be eliminated. If this condition never occurs we can keep the term.

This method is amazingly versatile. It is independent of the number of loops inside the diagram, or inside the propagator. It is also independent of the number of external legs. In addition it is rather fast. We tried it out on the sum of all 169788 5-loop gluon propagator diagrams in the background gauge. With the old topology matching and some tricks by which we could avoid having to match 4-loop subtopologies explicitly, the execution time was $507 \mathrm{sec}$. With the new method it went down to $106 \mathrm{sec}$. In addition the code is only 38 lines instead of 127 lines. The number of remaining diagrams was reduced to 80064 .

\section{Topologies}

As mentioned, the id,all was actually intended for manipulating topologies. Here is an example of determining all automorphisms of the nonplanar topology for 3-loop propagator graphs:
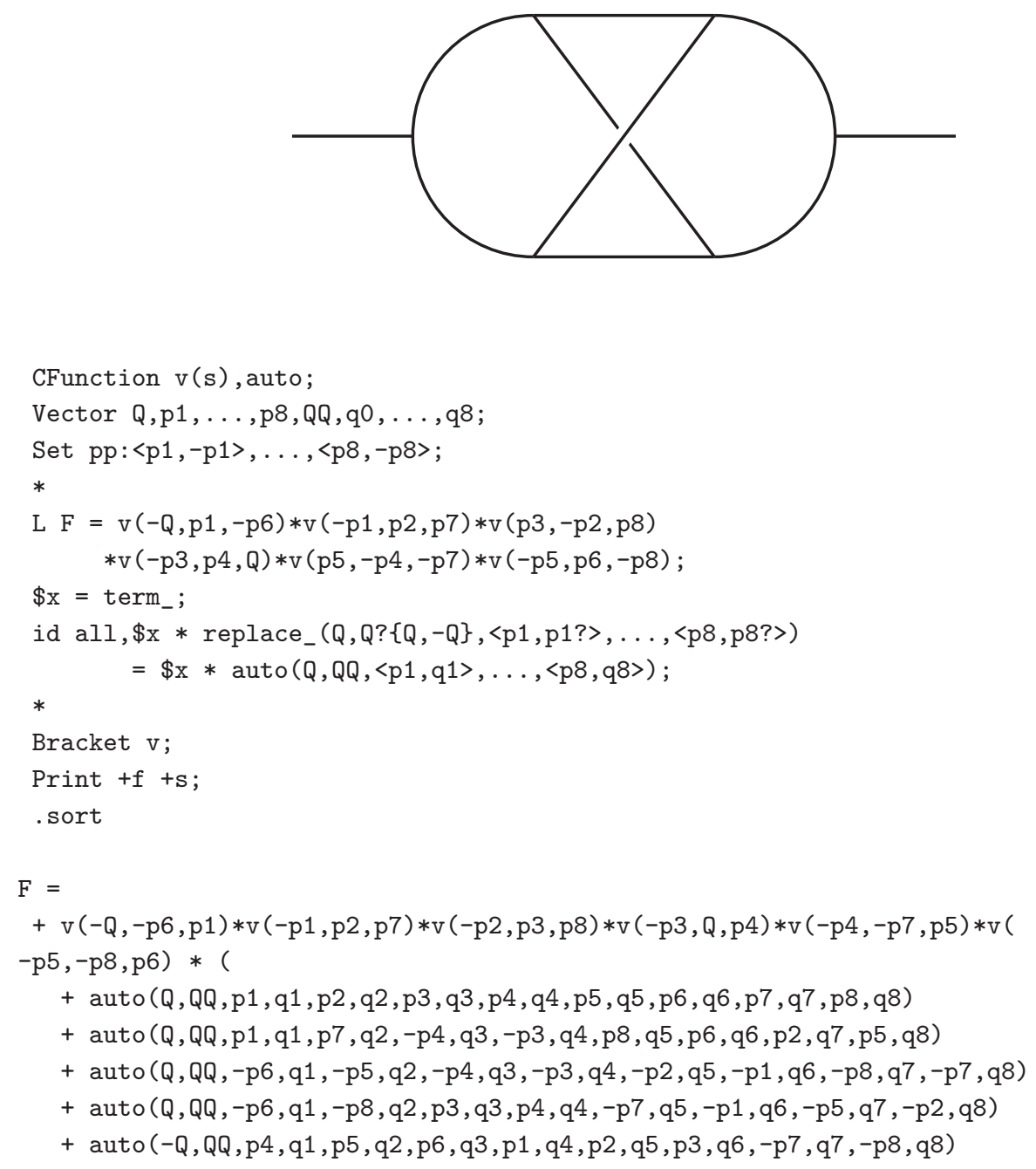


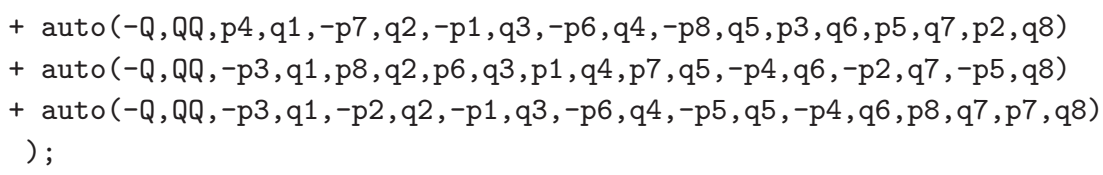

The dollar variable makes a copy of the current term and then the id statement matches the term onto itself, but thanks to the replace statement all variables have become wildcards and hence the statement generates all possible relabellings of the momenta. These relabellings are then stored in the function auto. The trick with the replace _ in the left hand side is due to Takahiro Ueda. It did not work right away as intended and needed a considerable amount of debugging, because in the original design this was never seen as a possibility.

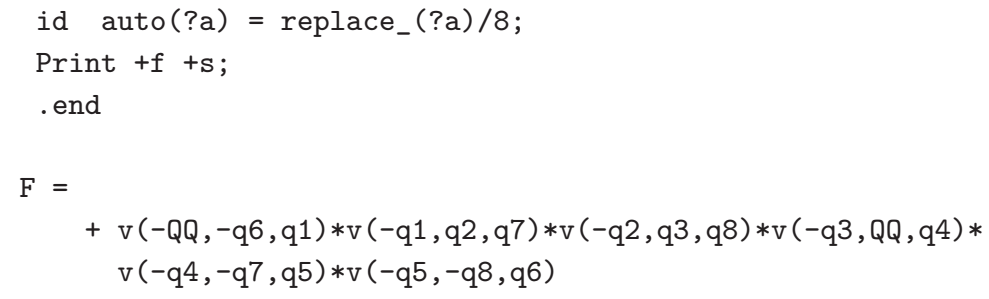

In the end we have the program execute those symmetry operations and we see that we get the input back, except for that now we have vectors q instead of vectors $\mathrm{p}$ (and QQ instead of Q).

As we saw, the id,all together with the trick to match the term with itself really hit the essence of graph automorphisms, and there was an immediate payoff in the form of the propagator example.

Now how do we determine the topology of a diagram in Form? And how do we transform to the notation that is being used in the library routines for those topologies? Again, in Mincer $[14,15,16]$ this could still be done 'by hand' because there were basically only 18 topologies. Here it is different with more than 400 topologies and if one would like to go to 5 loops there are thousands.

The first step is that the program that (automatically) produces the library routines should tell us what notation it uses. In the case of the Forcer library this is written into a file notation.h which looks like (just some representatives):

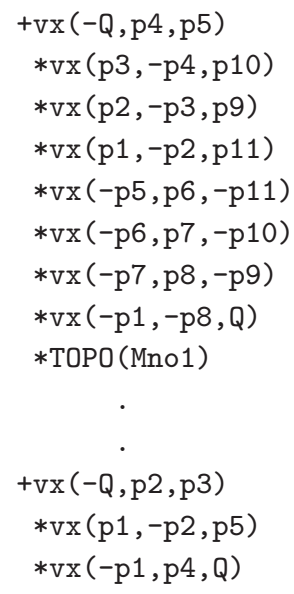


$* \mathrm{vx}(-\mathrm{p} 3,-\mathrm{p} 4,-\mathrm{p} 5)$

$* \operatorname{ex}(\mathrm{p} 2, \mathrm{p} 4)$

$* \mathrm{TOPO}($ Mt1star24)

These are those two topologies:
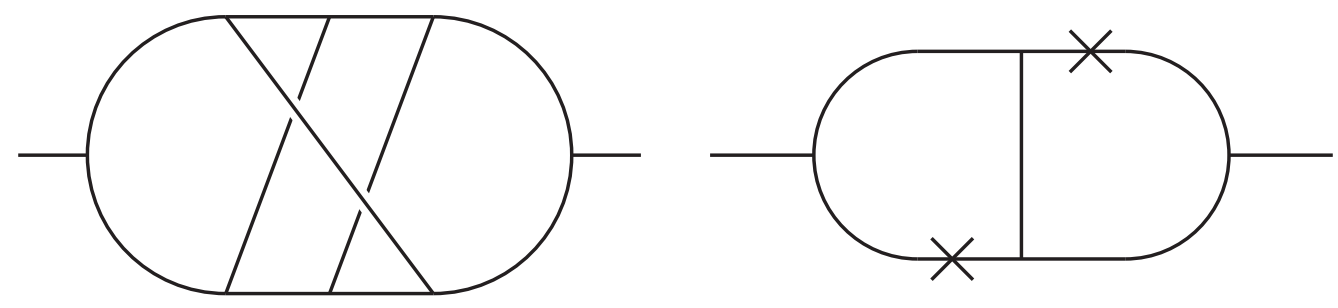

Each topology is represented by its vertices and momenta with a direction and a number. In addition there is a function ex of which the argument(s) tell us about insertions and of course the name of the topology. In the sequel we will ignore optimizations and just look at the main mechanism.

Step 1 is to make copies of the topologies into an array of $\$$-variables. We keep there only the vertices with momenta (as in example 1) and we strip the signs of the momenta (for speed). We also keep the ex function but change it to the symmetric function EX. We change the function vx to the symmetric function $v$. We also put the names of the topologies (we represent them by symbols) in a set tnames. Hence, if the first topology above becomes topology number 2 we have

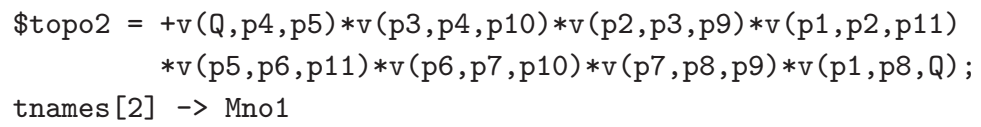

Next we create a dictionary.

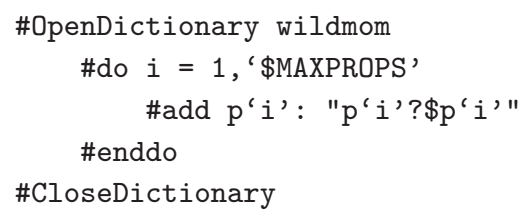

Originally the dictionaries were designed for creating complicated outputs. Their first use was for the GRACE [17] system. The feature we use here is that they print the output for a given variable as indicated in the text string. When this dictionary is active the system prints

$$
\mathrm{V}(\mathrm{Q}, \mathrm{p} 4 ? \$ \mathrm{p} 4, \mathrm{p} 5 ? \$ \mathrm{p} 5)
$$

instead of

$$
\mathrm{v}(\mathrm{Q}, \mathrm{p} 4, \mathrm{p} 5)
$$

Now we are ready for the main part. We start with making a copy of all our vertices and propagators into the function acc. Inside acc we strip the particle information and bring everything to a similar notation as the topologies in the dollar variables. The code becomes: 


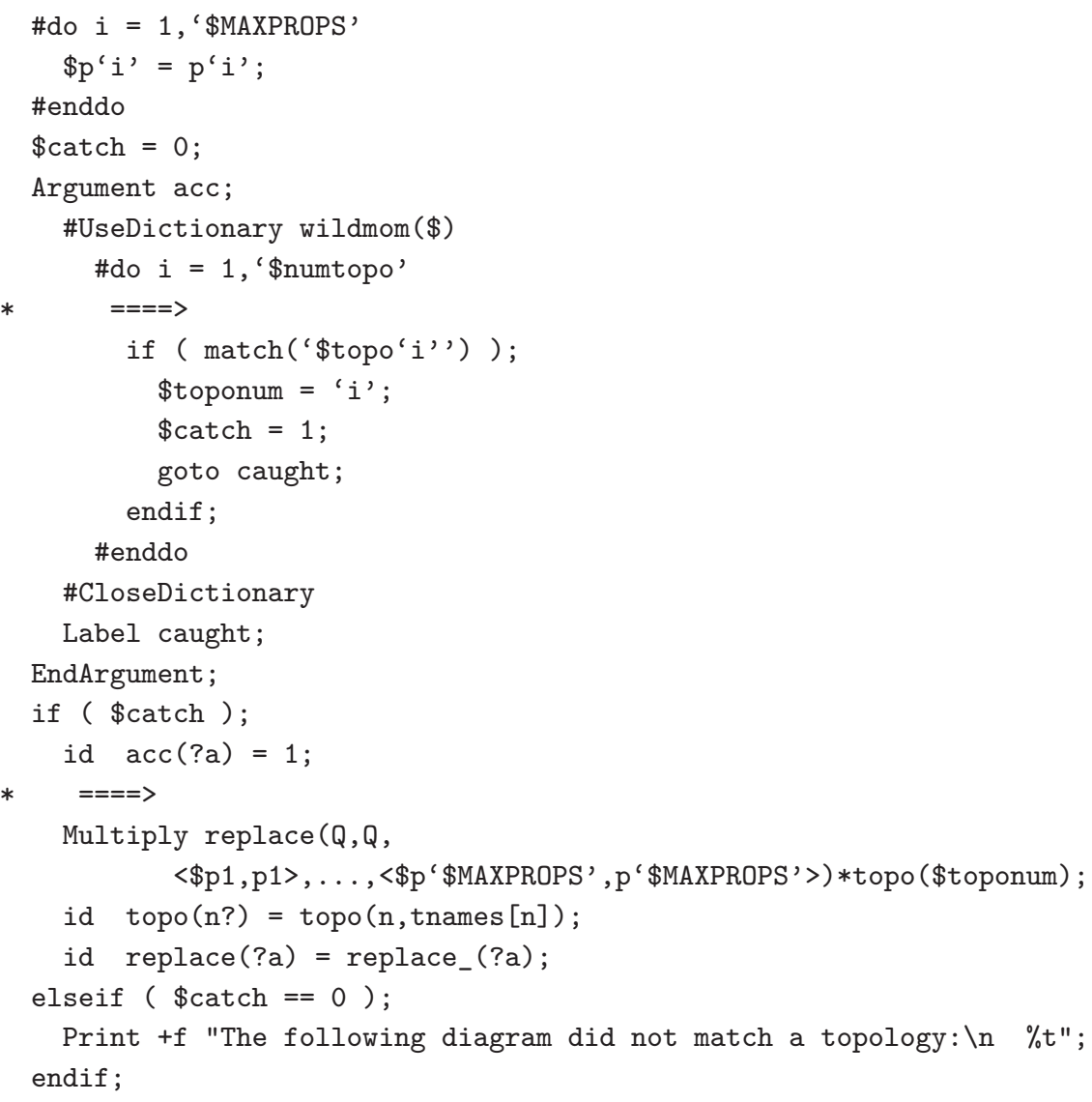

The key statements follow the arrows in the commentary. In the first we try to match a topology into the diagram. If this is successful, the values of the wildcards are loaded into the dollar variables $\$ \mathrm{p} 1, \$ \mathrm{p} 2, \ldots$ In the other statement we replace the values of the dollar variables in the diagram by the corresponding values in the topology.

As it is, when there are $N$ diagrams and $M$ topologies this needs on average $\frac{N M}{2}$ pattern matchings. This can be improved a lot by extra functions that tell how many 3-point and 4-point vertices there are and how many of those connect to an outside line. This is of course a type of hashing. We will skip that here.

After the topology has been determined, it is relatively easy to restore the signs on the momenta by comparing vertices. We skip that as well.

\section{Tables of statements}

The new feature here is the id_ function. But to explain what it does and why it was introduced is quite a story.

- Forcer uses dotproducts in the numerator, just like Mincer.

- LiteRed $[18,19]$ uses a clever choice of 15 invariants.

Why do we do this differently? 
- Reductions like the triangle rule are much more complicated in terms of invariants.

- The choice of invariants or dotproducts in the numerator can influence the complexity of the difficult reductions very much.

- Integrating out a one loop subdiagram is much easier with dotproducts.

The use of the dotproducts gives much more freedom in the choice of variables and hence more compact reductions. (See also the talk by Takahiro Ueda in these proceedings [1]). This goes at the cost of needing frequent rewritings between the various reduction steps. (You win some, you loose some).

Let us consider the no1 topology and what happens if the reduction removes a line.

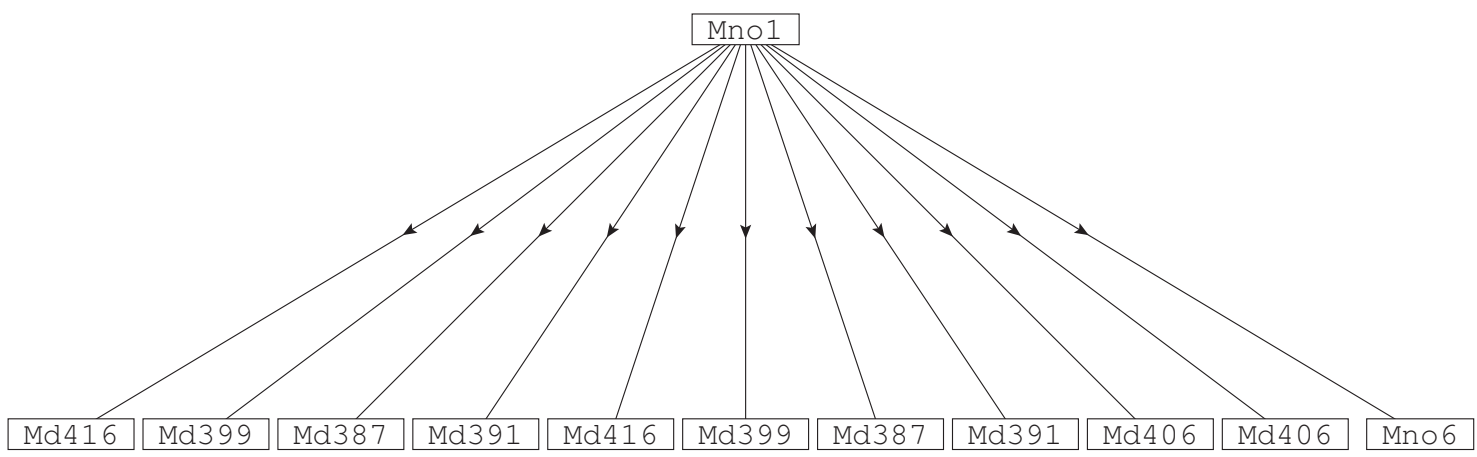

Each of the new topologies has its own notation and its own 'irreducible' dotproducts in the numerator. Hence there needs to be a relabelling. This can be done in a relatively simple way by a replace_function and in the ideal case one does this with a table. If all topologies have a number some table elements could look like

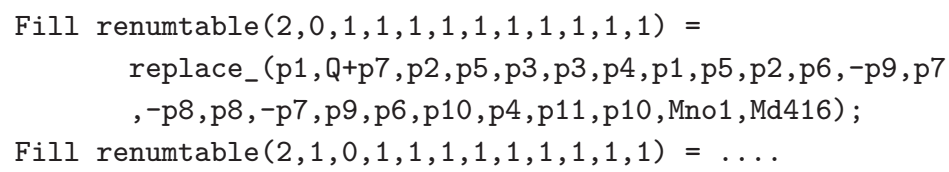

and after the no1 reductions have been done one can use the statement

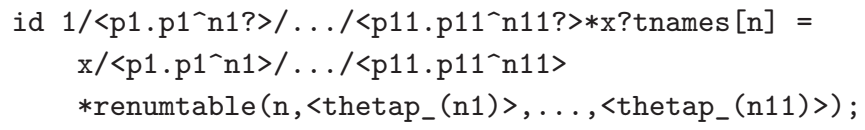

If the table is created by the (python) program that builds the whole system and if all table elements that are undefined are considered to be zero (which is the case if too many lines are missing) one needs only 14396 table elements (and not $2^{11} \times 400+$ ).

The above relabels the momenta. Now comes the tricky part. The dotproducts in the numerator may not be the dotproducts we need in the new topology. The bad part is that there can be high powers of those dotproducts when you want Andreas Vogt to calculate some nice large N Mellin moments in DIS $[9,10]$. Leaving that problem (it IS a big problem) aside we still have the case that we have a number of topologies that all need their numerators to be rewritten. And we would like to do this for all of them at the same time and with as few pattern matchings and if statements as possible.

For the high powers with many terms on the right hand side (rhs) we want to do 'slow substitutions' as in 


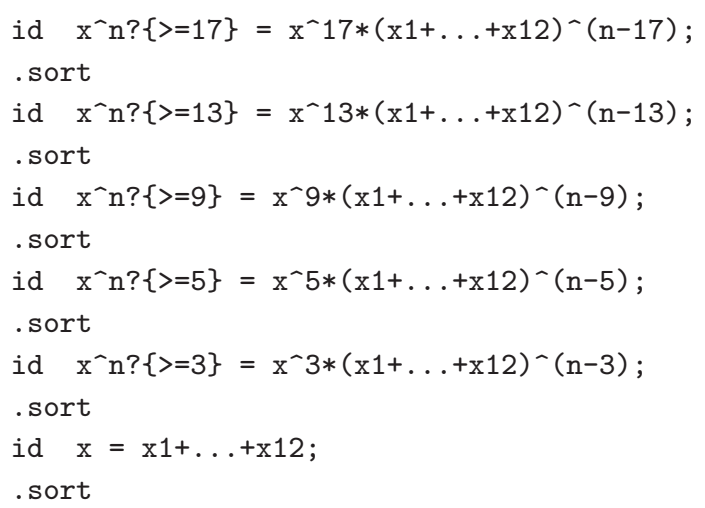

This way there are occasional combinations of identical terms and the whole process is usually much faster than a direct

id $\mathrm{x}=\mathrm{x} 1+\ldots+\mathrm{x} 12$;

.sort

Hence we want to set up such a system, but for each of the new topologies this may be different, and we will have several of such variables. One way would be

- Do this for the first topology, first variable.

- Do this for the first topology, second variable.

- Do this for the first topology, third variable.

- Do this for the first topology, fourth variable.

- Do this for the second topology, first variable.

- etc.

This gives an enormous number of steps and in addition for each step all other topologies are spectators that have to be carried around. Hence we would like to execute this all in parallel. But the number of steps for each variable is not a fixed number.

To do this optimally we have a special function id_. It looks a bit like replace_ but it has extra powers:

id_(p1.p2^17,p1.p2^17,p1.p2,p1.p1/2+p2.p2/2-p3.p3/2,p4.p5, $\mathrm{p} 4 \cdot \mathrm{p} 4 / 2+\mathrm{p} 5 \cdot \mathrm{p} 5 / 2-\mathrm{p} 6 \cdot \mathrm{p} 6 / 2)$

of

will be replaced during execution, after the term has been normalized, by the equivalent

id $\mathrm{p} 1 \cdot \mathrm{p} 2 \wedge 17=\mathrm{p} 1 \cdot \mathrm{p} 2 \wedge 17$;

al $\mathrm{p} 1 \cdot \mathrm{p} 2=\mathrm{p} 1 \cdot \mathrm{p} 1 / 2+\mathrm{p} 2 \cdot \mathrm{p} 2 / 2-\mathrm{p} 3 \cdot \mathrm{p} 3 / 2$;

al $\mathrm{p} 4 \cdot \mathrm{p} 5=\mathrm{p} 4 \cdot \mathrm{p} 4 / 2+\mathrm{p} 5 \cdot \mathrm{p} 5 / 2-\mathrm{p} 6 \cdot \mathrm{p} 6 / 2$;

which is to say: the first 17 powers of $\mathrm{p} 1 . \mathrm{p} 2$ are kept out from the further substitutions and then the other powers are replaced and also p4.p5 is replaced (we assume that there are fewer than 34 powers of $\mathrm{p} 1 . \mathrm{p} 2)$. The power is that this way we can let the program 
determine by itself what constitutes a good substitution scheme for each topology and then work out that scheme. It is this kind of AI that is eventually needed if we ever want to go even further.

We determine for each variable that needs to be replaced the maximum power. If the maximum power of $\mathrm{x}$ were to be 20 , and if there would already be 5 table elements from another variable we produce the statements

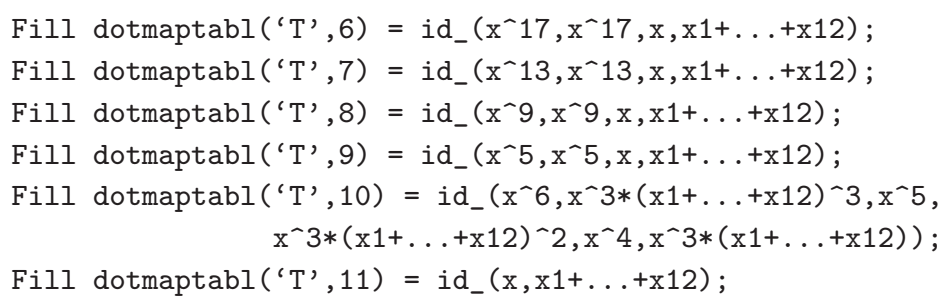

in which $\mathrm{T}$ defines the topology. We have a procedure that does this automatically but it is too unreadable to be shown here even though it takes only 17 lines. This way we define a table with statements waiting to be executed. If $\$ M$ is the maximum value of all the table elements for each topology we can now execute all statements with

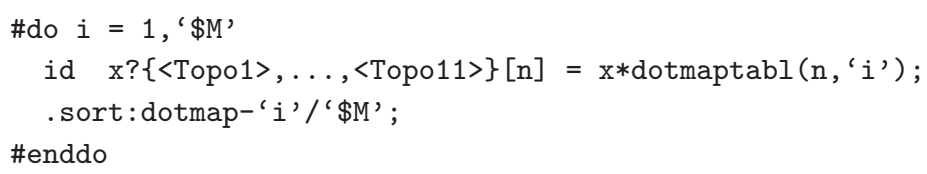

assuming that undefined table elements are replaced by one (new option). This executes the different statements for the different topologies in tandem each optimized for its topology and its maximum power and we have the optimal number of modules.

\section{Expansions}

As many people here have experienced, IBP reductions involving many loops can lead to extremely complicated rational polynomials for the coefficients. This is the same in Forcer. With the high complexities of some of our integrals we have run in polynomials involving $\epsilon^{500}$ and worse.

In Mincer we could expand and because Mincer does not have spurious poles, six powers in $\epsilon$ were usually enough. We did not manage to make Forcer free of spurious poles, and it may even be impossible. In such a case the safest solution is to work with (exact) rational polynomials, but these can become 'demanding': At $\mathrm{N}=4$ (Mellin moment) for the coefficient functions in DIS to four loops already a maximum term size of $140 \mathrm{~K}$ was not enough for some diagrams. The solution is to offer further possibilities: changing from exact to expansion to a predefined depth. What is a sufficient depth can be extrapolated from lower values of $\mathrm{N}$.

Example: The declaration

PolyRatfun rat;

would define exact rational polynomial coefficients in the function rat. At a later stage one could switch to 
PolyRatfun rat (expand, ep, 14);

after which Form would expand the rational polynomial to 14 terms deep, counting from the leading term in $\epsilon$ or $1 / \epsilon$. After this it would continue in this mode.

There is also a mode in which FORM can keep just the leading term and give it one for its coefficient. This is rather fast and allows one to determine what would be a proper depth of expansion.

There is a caveat: For the complicated terms this is much faster, because the rational calculus is now replaced by a normal 'addition', but since most terms have rather simple fractions, the expansion may make them more complicated and hence in the case of higher moments this mode could lead to slower programs. The optimal way (=when to change mode) to use this is still under investigation.

Probably the most efficient would be to have an 'absolute' depth of expansion that is based on the topology of the term. As one may imagine, this would be extremely complicated to implement. Hence this is just in the thinking stage. Suggestions are welcome.

\section{Conclusions}

We have seen a number of new Form features to make it easier to manipulate diagrams, notations and rewrites. This is not necessarily simple.

The new programs have already been used for some rather involved calculations in DIS $[9,10]$ that go considerable beyond the existing literature.

Another driving force behind this is to give Fonm more and more capabilities that allow the user to write ever smarter programs. Programs that can write parts of themselves, conditioned by the problem they are solving. The drawback of this is that the creator of such programs will have to think a bit more. The payoff is a program that can go beyond what was possible before.

Figures were made with Axodraw2 [20].

\section{References}

[1] T. Ueda, B. Ruijl and J.A.M. Vermaseren, Forcer: a FORM program for 4-loop massless propagators in these proceedings.

[2] Paulo Nogueira, , Automatic Feynman graph generation, J.Comput.Phys. 105 (1993) 279-289

[3] P.A. Baikov and K.G. Chetyrkin, Four Loop Massless Propagators: An Algebraic Evaluation of All Master Integrals, Nucl.Phys. B837 (2010) 186-220, arXiv:1004.1153.

[4] R.N. Lee, A.V. Smirnov and V.A. Smirnov, Master Integrals for Four-Loop Massless Propagators up to Transcendentality Weight Twelve, Nucl.Phys. B856 (2012) 95-110, arXiv:1108.0732.

[5] J.A.M. Vermaseren, New features of FORM, math-ph/0010025.

[6] J. Kuipers, T. Ueda, J.A.M. Vermaseren, J. Vollinga, FORM version 4.0, Comput.Phys.Commun. 184 (2013) 1453-1467, arXiv:1203.6543. 
[7] S.A. Larin, Paulo Nogueira, T. van Ritbergen, J.A.M. Vermaseren. The Three loop QCD calculation of the moments of deep inelastic structure functions, Nucl.Phys. B492 (1997) 338-378, hep-ph/9605317

[8] S. Moch, J.A.M. Vermaseren and A. Vogt, The Three-Loop Splitting Functions in QCD: The Helicity-Dependent Case, Nucl.Phys. B889 (2014) 351-400, arXiv:1409.5131.

[9] B. Ruijl, T. Ueda, J.A.M. Vermaseren, J. Davies and A. Vogt, First Forcer results on deep-inelastic scattering and related quantities, arXiv:1605.08408.

[10] J. Davies, A. Vogt, S. Moch, J.A.M. Vermaseren. Non-singlet coefficient functions for charged-current deep-inelastic scattering to the third order in QCD arXiv:1606.08907.

[11] C. Studerus, Reduze - Feynman integral reduction in $C++$, Comput. Phys. Commun. 181 (2010) 1293-1300, arXiv:0912.2546.

[12] A. von Manteuffel and C. Studerus, Reduze 2 - Distributed Feynman Integral Reduction, arXiv: 1201.4330

[13] Paulo Nogueira, Abusing qgraf, Nucl.Instrum.Meth. A559 (2006) 220-223.

[14] S.G. Gorishnii, S.A. Larin and F.V. Tkachov, INR preprint P-0330 (Moscow, 1984).

[15] S.G. Gorishnii, S.A. Larin, L.R. Surguladze and F.V. Tkachov, Mincer: Program for Multiloop Calculations in Quantum Field Theory for the Schoonschip System, Comp. Phys. Comm. 55 (1989) 381.

[16] S.A. Larin, F.V. Tkachov and J.A.M. Vermaseren, The FORM version of MINCER, NIKHEF-H-91-18.

[17] P.H. Khiem at al. Full $\mathcal{O}(\alpha)$ electroweak radiative corrections to $e^{+} e^{-} \rightarrow e^{+} e^{-} \gamma$ at the ILC with GRACE-Loop, Phys. Lett. B740 (2015) 192-198, arXiv:1403.6557.

[18] R.N. Lee. Presenting LiteRed: a tool for the Loop InTEgrals REDuction. arXiv:1212.2685.

[19] Roman N. Lee, LiteRed 1.4: a powerful tool for reduction of multiloop integrals. J.Phys.Conf.Ser. 523 (2014) 012059.

[20] John C. Collins and J.A.M. Vermaseren, Axodraw Version 2, arXiv:1606.01177. 